\title{
Energy and economic analysis of thermoelectric generator on wood fuel
}

\author{
Oleg Marchenko \\ Melentiev Energy Systems Institute of Siberian Branch of the Russian Academy of Sciences, Irkutsk, Russia
}

\begin{abstract}
The advantages of thermoelectric generator (TEG) are the absence of moving parts, reliability, long service life, the possibility of fully automatic operation without maintenance. At the same time, TEGs also have disadvantages - low efficiency and high cost. In this regard, it is advisable to use them in autonomous installations of small power, as well as in devices intended mainly for heat supply, when the low efficiency of thermal energy conversion into electrical energy does not play a role. It is the latter case that is realized in furnaces that burn biomass. In this paper, we consider the main physical effects due to which the direct conversion of thermal energy into electrical energy occurs in semiconductor thermoelements. The conjugate problem of heat and electric charge transfer with temperature-dependent properties of a semiconductor is reduced to a problem with constant properties. Based on the literature data, the boundary conditions for the TEG were selected, its sizes were optimized, current-voltage characteristics were constructed, and the maximum power and efficiency were determined. Estimates of the cost and economic efficiency of using TEG are made.
\end{abstract}

\section{Introduction}

Most technologies for using wood biomass for power supply to consumers include the stages of thermal and then mechanical energy production. Mechanical energy is converted into electrical energy in electromechanical generators.

There are technologies that make it possible to obtain electrical energy without preliminary production mechanical energy. One of these technologies is the direct conversion of thermal energy into electrical energy in thermoelectric generators (TEG) $[1,2]$. It uses thermoelectric effects: $a$ ) the temperature gradient in a solid causes diffusion of the electric charge carriers (in a semiconductor - electrons and holes), i.e. electric current; $b$ ) electric current is accompanied by heat generation or absorption.

The main thermoelectric effects - Seebeck, Peltier and Thomson - were discovered in the XIX century. Subsequently, it turned out that TEG of metal thermocouples have too low efficiency. At the same time, the use of semiconductor materials, including those with a variable doping profile or composed of separate segments, each optimized for the corresponding temperature range, made it possible to achieve an efficiency of up to $12-15 \%$ [3].

TEGs do not have moving parts, can operate without maintenance for a long time, they are compact, reliable, noiseless and environmentally-friendly power sources. At the same time, TEGs have disadvantages - high cost and low efficiency. These features have identified the application areas of TEG: space and ground power plants of low power, including those with nuclear heat sources (reactors and radioisotope sources). Such installations are designed for long-term operation without maintenance and therefore more important characteristics than efficiency are reliability, weight, specific energy content, specific power, etc.

Other options for using low-efficiency power sources are for electrochemical protection of gas pipelines against corrosion when fuel is available in excess [3, 4], or for heat recovery, which is normally lost in the surrounding space [5]. For example, TEG designs have been developed for utilizing heat from exhaust gases from cars $[6,7]$ or heat that is lost during combustion or gasification of biomass $[8,9]$.

Thermoelectric generators make it possible to create new commercially attractive systems that use waste heat and renewable energy resources $[10,11]$. Wood biomass is an important source of renewable energy, the use of which in the global energy sector is increasing. Utilization of waste from industrial timber harvesting and its processing through energy use has a positive effect on the environmental situation and in some cases allows increasing the reliability and efficiency of heat and power supply to consumers. Combining the TEG with biomass stoves allows the generation of electrical energy using waste heat.

The systems proposed for this in the literature [1214] are designed mainly for the use of standard thermoelectric modules with a fixed design. It is of interest to evaluate the ultimate characteristics of such devices that can be achieved by optimizing their design. The purpose of this work is the formation of a

$\overline{\text { E-mail address: } \text { marchenko@,isem.irk.ru }}$ 
mathematical model of TEG that recovers heat generated during the combustion of biomass, the choice of boundary conditions for the equations of heat and electric charge transfer, the calculation of energy and economic characteristics, and the optimization of the TEG design.

\section{Design of semiconductor power sources}

A thermoelectric generator is a solid state heat engine that uses electrons and holes as a working fluid to directly convert thermal energy into electrical energy. Thermoelectric generators consist of $\mathrm{p}$ - and n-types semiconductor legs joined into thermoelements (thermocouples) by metal plates (Fig. 1). The thermoelements are thermally and electrically insulated from their surroundings. The heat flux passes through the thermoelements from the hot junction to the cold one. The electrical energy generated from the thermal energy does work on an external load; the remainder of the thermal energy is rejected from the cold junction.

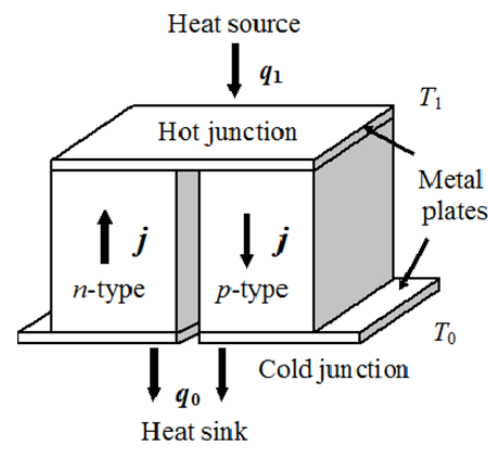

Fig. 1. Thermoelement consisting of two semiconductor legs (p-and n-type): $\boldsymbol{q}$ is the heat flux density, $T$ is the temperature, $j$ is the electric current density; subscripts: $0-$ cold junction, 1 - hot junction.

Several thermoelements form thermoelectric batteries which can be used both separately and be connected with one another in series-parallel electrical and thermal circuits (Fig 2). Heat conductors simultaneously serve as electrical insulators, preventing the short circuit through the framework. Thermal insulation reduces parasitic heat transfer between the heat source and the heat sink, and also protects the semiconductor alloy from evaporation and chemical interactions at high temperatures.

A thermoelectric generator consists of a set of thermoelectric batteries, a heat source and a cooling system. The manufacturing of the TEG from separate interchangeable batteries makes it possible to simplify assembly, control the generator quality and increase its reliability.

Liquids or gases can be used to transfer heat to TEG hot junctions and to remove heat from cold junctions. Fig. 3 represents one of the variants of a space power plant with a TEG [2].

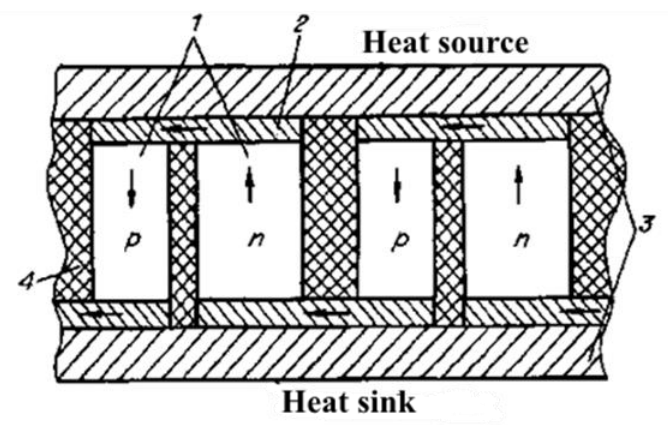

Fig. 2. Thermoelectric battery: 1 - semiconductor legs, $2-$ metal buses, 3 - heat conductor, 4 - heat insulation.

\section{Thermoelectric phenomena}

In an unevenly heated continuous medium, in addition to heat flux the temperature difference causes diffusion of the electric charge carriers, i.e. electric current. Electric current in turn is accompanied by heat generation or absorption.

The dependences of the electric current density $\boldsymbol{j}$ and heat flux density $\boldsymbol{q}$ on the temperature gradients $T$ and potential $\varphi$ have the following form [15]:

$$
\begin{gathered}
\boldsymbol{j}=-(1 / \rho)(\alpha \nabla T+\nabla \varphi) \\
\boldsymbol{q}=-\kappa \nabla \mathrm{T}+\alpha \mathrm{T} \boldsymbol{j}
\end{gathered}
$$

where $\alpha$ is the thermoelectric power (Seebeck coefficient), $\kappa$ is the thermal conductivity and $\rho$ is the electrical resistivity. Thermoelectric material properties $\alpha, \kappa$ and $\rho$ depend on temperature and coordinate.

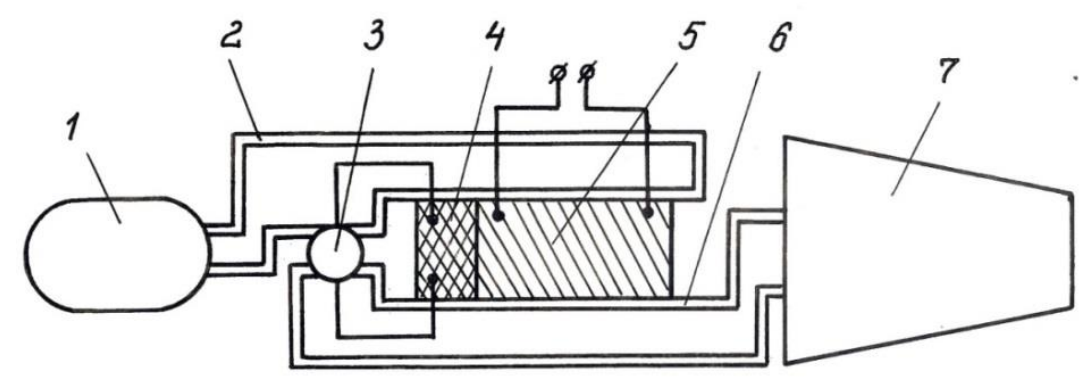

Fig. 3. Diagram of a power plant with a thermoelectric generator: 1 - heat source (nuclear reactor), 2 - hot fluid, 3 - pump, 4 - TEG pumping section, 5 - TEG main section, 6 - cold fluid, 7 - radiator. 
In steady state conditions, the governing equations result from the principles of conservation of charge and energy [15]

$$
\begin{gathered}
\nabla \boldsymbol{j}=0 \\
\nabla(\boldsymbol{q}+\varphi \boldsymbol{j})=0
\end{gathered}
$$

Solving differential equations (3) and (4) with the appropriate boundary conditions, one can find the temperature and potential distributions, and then determine the heat flux through the considered surface $S$

$Q=\int_{S}(\boldsymbol{q} \boldsymbol{n}) d S$

electric current

$J=\int_{S}(\boldsymbol{j n}) d S$

and other characteristics. Here $\boldsymbol{n}$ is the outward normal.

\section{Energy characteristics thermocouple legs}

Let us assume that $a$ ) the semiconductor leg is homogeneous and isotropic, $b$ ) the problem is static and one-dimensional and $c$ ) thermoelectric material properties $\alpha, \kappa$ and $\rho$ are constant (independent of temperature).

With constant material properties, the onedimensional conjugate problem of heat and electric charge transfer has an exact analytical solution. From system (1) - (4) with boundary conditions

$$
\begin{cases}T(0)=T_{0}, & T(l)=T_{1} \\ \varphi(0)=\varphi_{0}, & \varphi(l)=\varphi_{1}\end{cases}
$$

where $l$ is the length of the thermoelement leg, there follow the dependence of the temperature on the coordinate $x(0 \leq x \leq l)$

$$
\begin{aligned}
T(x)=T_{0}+\left(T_{1}-\right. & \left.T_{0}\right)(x / l)+ \\
& +(1 / 2)\left(j^{2} \rho / \kappa\right)(l-x) x
\end{aligned}
$$

heat flux density at the boundaries $x=0$ and $x=l$ (heat balance equations)

$$
\begin{aligned}
q_{0} & \equiv q(0)=[\kappa d T / d x+j \alpha T]_{x=0}= \\
& =\kappa\left(T_{1}-T_{0}\right) / l+j \alpha T_{0}+(1 / 2) j^{2} \rho l \\
q_{1} & \equiv q(l)=[\kappa d T / d x+j \alpha T]_{x=l}= \\
& =\kappa\left(T_{1}-T_{0}\right) / l+j \alpha T_{1}-(1 / 2) j^{2} \rho l
\end{aligned}
$$

dependence of the potential difference (voltage) $V$ on the current density $j$, i.e. volt-ampere characteristic

$$
\varphi_{0}-\varphi_{1} \equiv V=\alpha\left(T_{1}-T_{0}\right)-j \rho l
$$

electric power

$$
w=j V=q_{1}-q_{0}
$$

and efficiency

$$
\eta=w / q_{1}
$$

Multiplying the specific values $q_{0}$ and $q_{1}$ by the cross-sectional area of the leg $S$, summing the heat fluxes and voltages, one can obtain a system of equations for an arbitrary TEG design, consisting of sequentially and parallel connected thermoelements legs, metal buses and heat conductors [3].

At constant junction temperatures $T_{0}$ and $T_{1}$, the specific power reaches the maximum value

$$
w_{\text {max }}=\alpha^{2}\left(T_{1}-T_{0}\right)^{2} / 4 \rho l
$$

at current

$$
j_{\text {opt }}=\alpha\left(T_{1}-T_{0}\right) / 2 \rho l
$$

Efficiency reaches its maximum value

$$
\eta_{\max }=\left[\left(T_{1}-T_{0}\right) / T_{1}\right]\left[(M-1) /\left(M+T_{0} / T_{1}\right)\right]
$$

at current

$$
j_{o p t}=\alpha\left(T_{1}-T_{0}\right) /(M+1) \rho l
$$

where

$$
M=\sqrt{1+Z\left(T_{1}+T_{0}\right) / 2}
$$

and

$$
Z=\alpha^{2} / \rho \kappa
$$

is figure of merit of thermoelectric material [1].

\section{Method of average parameters}

The properties of almost all thermoelectric materials change in the temperature range from $T_{0}$ to $T_{1}$ so much that it is impossible to neglect their temperature dependence. For an example Fig. 4 presents the characteristics of the widely-spread bismuth telluride $\left(\mathrm{Bi}_{2} \mathrm{Te}_{3}\right)[16,17]$.

With variable properties of materials, the small value of thermoelectric effects in comparison with the heat transferred by thermal conductivity makes it possible to find a solution to the problem by the method of perturbation. The perturbation method already in the first approximation provides an accuracy acceptable for practice: the error does not exceed $1 \%$. In this case, it turns out that the solution for variable properties of materials practically coincides with the solution given above for constant properties with their replacement by properties averaged over the temperature range by integration [18]

$$
\left\{\begin{array}{c}
\bar{\alpha}=\frac{1}{T_{1}-T_{0}} \int_{T_{0}}^{T_{1}} \alpha(T) d T \\
\bar{\kappa}=\frac{1}{T_{1}-T_{0}} \int_{T_{0}}^{T_{1}} \kappa(T) d T \\
\bar{\rho}=\overline{\rho \kappa} / \bar{\kappa}
\end{array}\right.
$$


(a)

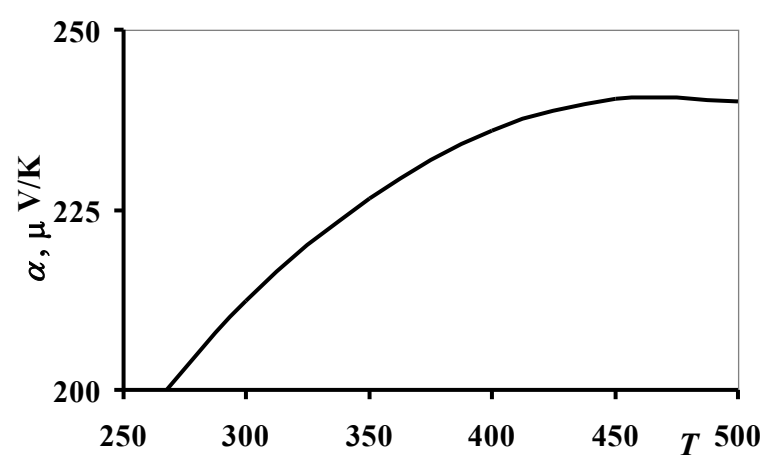

(b)

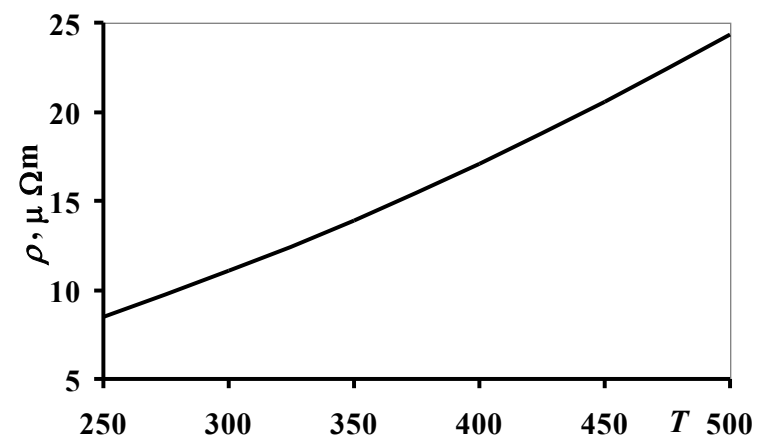

(c)

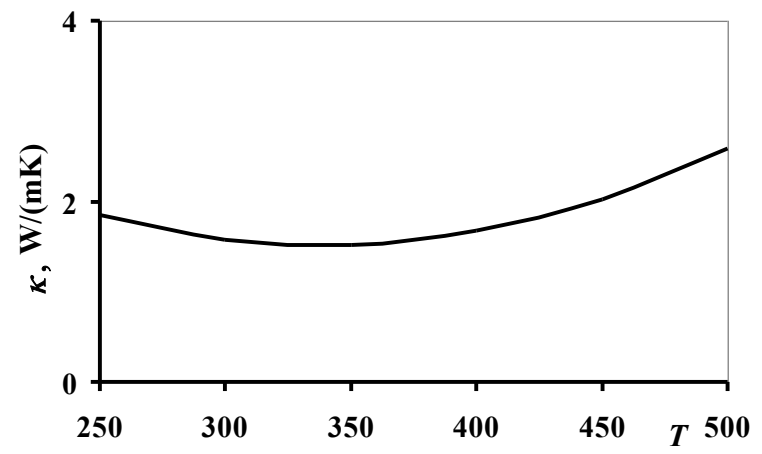

(d)

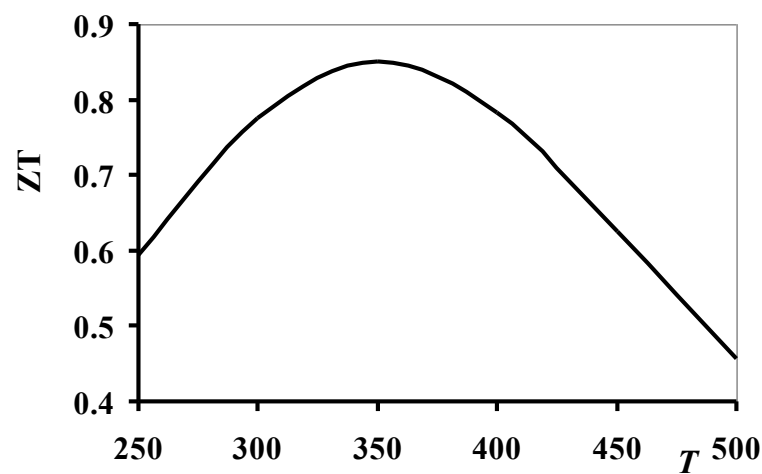

Fig. 4. Temperature dependence of $\mathrm{Bi}_{2} \mathrm{Te}_{3}$ properties: Seebeck coefficient $(a)$, electrical resistivity $(b)$, thermal conductivity (c) and dimensionless figure of merit $Z T=\left(\alpha^{2} / \rho \kappa\right) T(d), T$ is temperature in $\mathrm{K}$.

\section{Mathematical model}

To simulate a TEG on wood fuel, we assume that its junctions exchange heat with a heat source and a cooling system, having temperatures $T_{H}$ and $T_{C}$ (Fig. 5) according to Newton's law (heat flux is proportional to the temperature difference). In this case, the junction temperatures do not remain constant and depend on the current. The system of equations for determining the temperatures of junctions and heat fluxes is:

$$
\begin{gathered}
q_{1}=\left(T_{H}-T_{1}\right) / \xi_{1} \\
q_{1}=\kappa^{*}\left(T_{1}-T_{0}\right) / l+j \alpha T_{1}-(1 / 2) j^{2} \rho^{*} l \\
q_{0}=\kappa^{*}\left(T_{1}-T_{0}\right) / l+j \alpha T_{0}+(1 / 2) j^{2} \rho^{*} l \\
q_{0}=\left(T_{0}-T_{\mathrm{C}}\right) / \xi_{0}
\end{gathered}
$$

where $\xi_{1}$ and $\xi_{0}$ are thermal resistances,

$$
\begin{aligned}
\kappa^{*} & =\kappa\left(1+\varepsilon_{\kappa}\right) \\
\rho^{*} & =\rho\left(1+\varepsilon_{\rho} l_{0} / l\right)
\end{aligned}
$$

and constants $\varepsilon_{\kappa}$ and $\varepsilon_{\rho}$ take into account heat leakage through thermal insulation and additional resistances of metal buses and contact electrical resistances $\left(l_{0}\right.$ is reference length) [3].

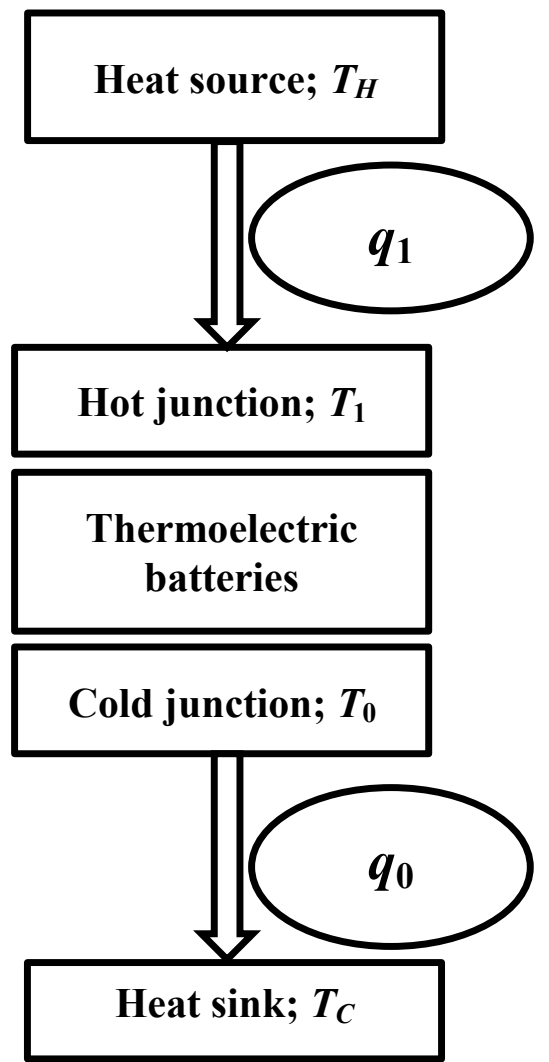

Fig. 5. TEG scheme.

An important characteristic of a TEG is its cost. For subsequent optimization of the TEG design, the dependence of the cost on the legs length can be approximated as follows: 


$$
p=p_{0}\left(1+\varepsilon_{p}\left(l / l_{0}-1\right)\right)
$$

This shows that $p=p_{0}$ for $l=l_{0}$ and $p=p_{0}\left(1-\varepsilon_{p}\right)$ for $l=$ 0 . Thus, $p_{0}$ is reference cost and $\varepsilon_{p}$ is the share of semiconductor material in the total cost.

\section{Initial data}

Calculations were carried out for TEG made of thermoelectric alloy bismuth telluride $\left(\mathrm{Bi}_{2} \mathrm{Te}_{3}\right)$ [19]. The main data for the mathematical model were selected on the basis of analysis and generalization of experimental and theoretical studies of similar devices [12-14, 20-22] (Table 1). Energy and cost characteristics are related to the unit of the TEG area.

Table 1. TEG model characteristics.

\begin{tabular}{llll}
\hline Characteristics & Value & Characteristics & Value \\
\hline$T_{H}, \mathrm{~K}$ & 750 & $\varepsilon_{\rho}$ & 0.06 \\
$T_{C}, \mathrm{~K}$ & 300 & $\varepsilon_{p}$ & 0.5 \\
$\xi_{0}, \mathrm{~cm}^{2} \mathrm{~K} / \mathrm{W}$ & 10 & $l_{0}, \mathrm{~cm}$ & 0.5 \\
$\xi_{1}, \mathrm{~cm}^{2} \mathrm{~K} / \mathrm{W}$ & 25 & $p_{0}, \$ / \mathrm{cm}^{2}$ & 2.5 \\
$\varepsilon_{K}$ & 0.05 & & \\
\hline
\end{tabular}

\section{Calculation results and their analysis}

The calculations were carried out for two variants.

In the first variant, with a constant length of thermoelement legs $l=0.5 \mathrm{~cm}$, the current $j$ was varied (Fig. 5). With increasing current, the hot junction temperature $T_{1}$ decreases, the cold junction temperature $T_{0}$ increases and the temperature difference across the semiconductor $\Delta T=T_{1}-T_{0}$ decreases from 202 to $135 \mathrm{~K}$ (see Fig. $5 a$ ). This is due to an increase in heat fluxes $q_{1}$ and $q_{0}$ and an increase in temperature drop across thermal resistances $\xi_{1}$ and $\xi_{0}$. The voltage decreases from the maximum value at $j=0$ to zero at a shortcircuit current of $36.5 \mathrm{~A} / \mathrm{cm}^{2}$ due to a decrease in the EMF and a voltage drop across the internal resistance of thermoelements (see Fig. 5b). Efficiency peaks at 17.1 $\mathrm{A} / \mathrm{cm}^{2}$, power at $18.1 \mathrm{~A} / \mathrm{cm}^{2}$ (see Fig. $5 c$ ). The difference in energy characteristics between these modes is negligible: the efficiency in the maximum power mode and the power in the maximum efficiency mode deviate from their maximum values of $5.3 \%$ and $4.3 \mathrm{~W} / \mathrm{cm}^{2}$ by no more than $0.5 \%$ (Table 2 ).

Table 2. Optimal solutions.

\begin{tabular}{llllll}
\hline Variant & $l$, & $j$, & $w$, & $\eta$, & $p / w$, \\
& $\mathrm{cm}$ & $\mathrm{A} / \mathrm{cm}^{2}$ & \multicolumn{6}{c}{$\mathrm{W} / \mathrm{cm}^{2}$} & $\begin{array}{l}\% \\
\text { l }\end{array}$ & $\$ / \mathrm{W}$ \\
\hline \multicolumn{7}{c}{ const } \\
$w=\max$ & 0.5 & 18.1 & 0.428 & 5.26 & 5.85 \\
$\eta=\max$ & 0.5 & 17.1 & 0.426 & 5.28 & 5.87 \\
\hline \multicolumn{6}{c}{$l=\mathrm{var}$} \\
$w=\max$ & 0.86 & 15.0 & 0.459 & 7.03 & 7.40 \\
$p / w=\min$ & 0.36 & 20.0 & 0.382 & 4.23 & 5.62 \\
\hline
\end{tabular}

(a)

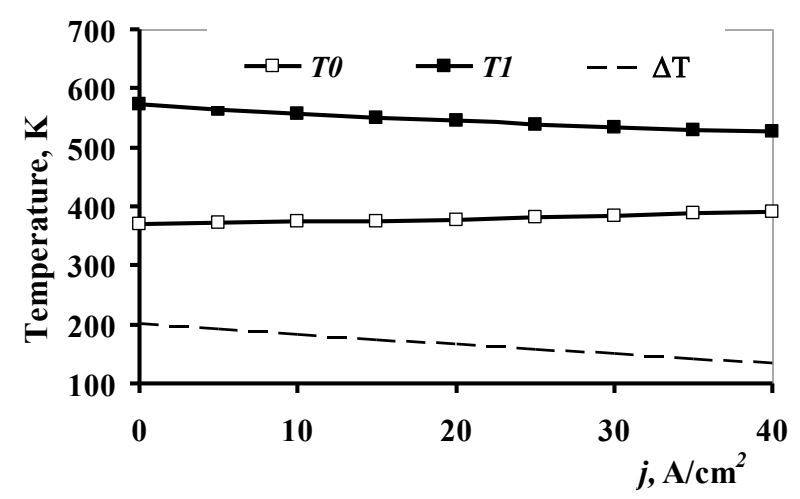

(b)

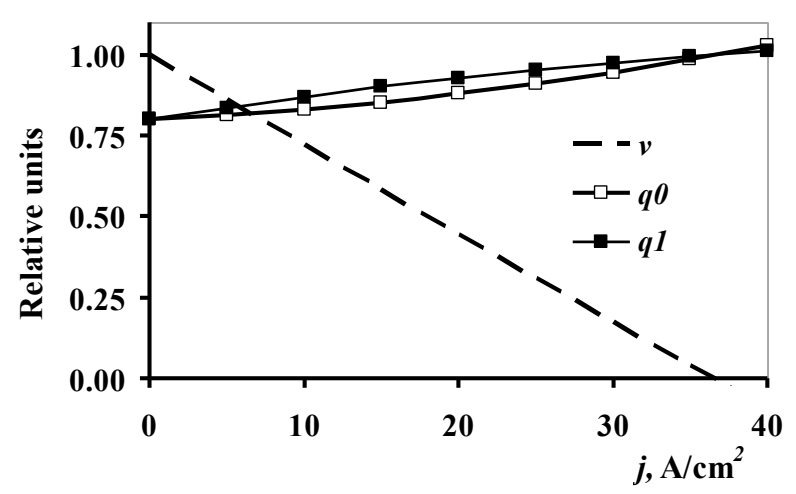

(c)

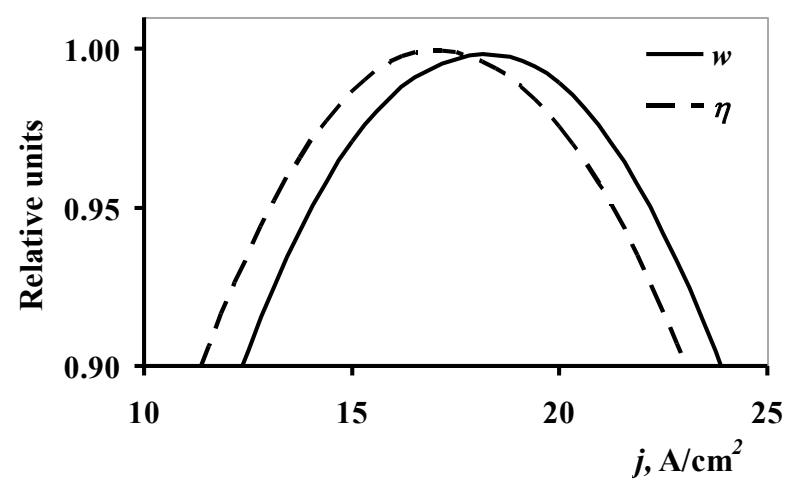

Fig. 5. Characteristics dependencies on current density: temperatures and temperature difference $\Delta T=T_{1}-T_{0}(a)$, voltage and heat flux density $(b)$, power density and efficiency (c).

In the second variant, the legs length $l$ was varied, and the current $j$ was chosen from the requirement of the maximum power $w$ (Fig. 6). With increasing legs length, the hot junction temperature $T_{1}$ increases, the cold junction temperature $T_{0}$ decreases, and the temperature difference across the semiconductor increases (see Fig. $6 a$ ). This is due to a decrease in heat fluxes $q_{1}$ and $q_{0}$ and a decrease in temperature drop across thermal resistances $\xi_{1}$ and $\xi_{0}$. The voltage increases due to the increase in temperature difference (see Fig. 6b). With an increase in $l$, the efficiency monotonically increases, the cost per 
unit of power reaches a minimum at $l=0.36 \mathrm{~cm}$, and the power reaches a maximum at $l=0.86 \mathrm{~cm}$ (see Fig. $6 c$ ). In this case, the difference between the modes $w=\max$ and $p / w=\min$ is significant: the deviation of characteristics at the transition from one mode to another is $20-30 \%$.

In the considered variants, the TEG on wood fuel provides a specific power up to $0.46 \mathrm{~W} / \mathrm{cm}^{2}$, an efficiency up to $7 \%$ and a specific cost of about $\$ 5.6 / \mathrm{W}$.

(a)

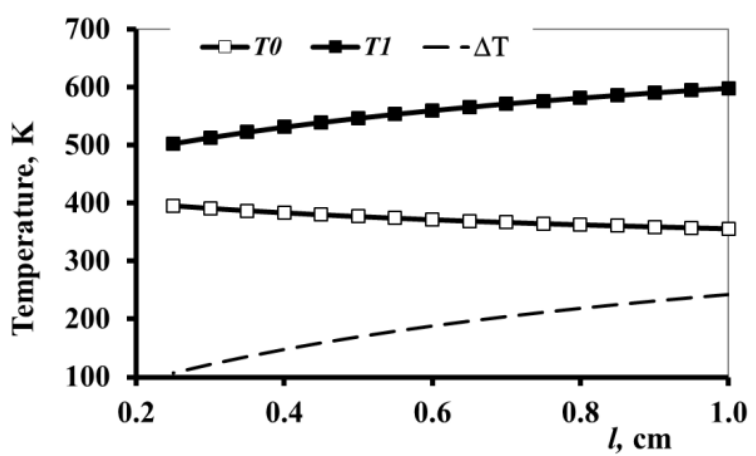

(b)

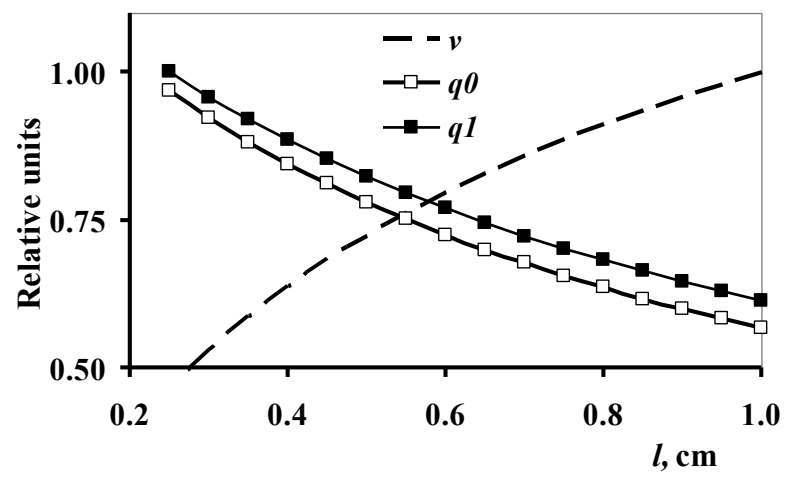

(c)

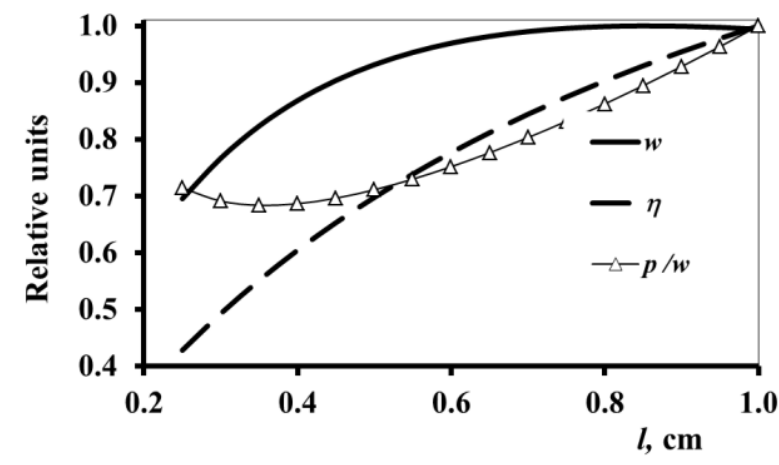

Fig. 6. Characteristics dependencies on thermocouple legs length: temperatures and temperature difference $\Delta T=T_{1}-T_{0}$ $(a)$, voltage and heat flux density $(b)$, power density, efficiency and unit power cost $p / w(c)$.

\section{Conclusion}

To assess the characteristics of TEG on woody biomass, a mathematical model was used in the form of a conjugate problem of heat and electric charge transfer in the thermoelement legs and a system of equations describing the heat exchange of TEG with the environment according to Newton's law.

With constant material properties, the onedimensional stationary problem for the thermoelement leg has an exact analytical solution. It is shown that, for temperature-dependent properties, the small value of thermoelectric effects in comparison with the heat transferred by thermal conductivity makes it possible to find a solution to the problem by the perturbation method. Already in the first approximation, the perturbation method provides an accuracy acceptable for practice: the error does not exceed $1 \%$. It turns out that this approximation is equivalent to using an analytical solution with constant properties, replacing them with properties averaged over the temperature range by integration.

Based on the analysis and generalization of experimental and theoretical studies of similar devices, the initial data for the mathematical model are selected the temperature of the heat source, the environment, as well as thermal resistance and other parameters.

Calculations were performed for two variants: $a$ ) with a constant length of the thermoelement legs and varying the current from zero to the short-circuit current and $b$ ) with varying the legs length choosing the current from the requirement of maximum power. The regularities of changes in temperatures, energy and cost characteristics are investigated. It is shown that at a constant length of the thermoelement legs, the modes of maximum power and maximum efficiency differ insignificantly. With an increase in the length of the legs, the cost per unit of power first reaches a minimum, then the power reaches a maximum, and the efficiency increases monotonically.

Under the considered conditions, the TEG on wood fuel provides a specific power up to $0.46 \mathrm{~W} / \mathrm{cm}^{2}$, an efficiency up to $7 \%$ and a specific cost of about $\$ 5.6 / \mathrm{W}$.

\section{Acknowledgement}

The reported study was funded by Russian Foundation for Basic Research (RFBR) № 19-58-80016; Department of Science and Technology of India (DST), №CRG/2018/004610, DST/TDT/TDP-011/2017; Ministry of Science and Technology of the People's Republic of China (MOST), №2018YFE0183600; National Research Council of Brazil (CNPq), №402849/2019-1; National Research Foundation of South Africa (NRF), № BRIC190321424123. Studies performed using equipment of the multi-access scientific center "High Temperature Circuit".

\section{References}

1. A.F. Ioffe, Semiconductor thermoelements and thermoelectric cooling (Infosearch Limited, London, 1957) 
2. D.M. Rowe (Ed.), CRC handbook of thermoelectrics (CRC Press, Boca Raton, FL, 1995)

3. O.V. Marchenko, A.P. Kashin, V.I. Lozbin, M.Z. Maksimov, Methods for performance calculation of thermoelectric generators (Nauka, Novosibirsk, 1995) [in Russian].

4. V.I. Yarygin, Ye.A. Meleta, J. of Thermoelectricity, 3, 95-103 (1998)

5. C. Wu, App. Therm. Eng., 16, 63-69 (1996)

6. S. Vale, L. Heber, P.J. Coelho, C.M. Silva, Energy Convers. Manage., 133, 167-177 (2017)

7. A. Marvão, P. J. Coelho, H.C. Rodrigues, Energy Convers. Manage., 179, 178-191 (2019)

8. H. Ishaq, S. Islam, I. Dincer, B. S. Yilbas, J. of Cleaner Production, Article 12062 (to be published)

9. H.-K. Ma, C.-P. Lin, H.-P. Wu, C.-H. Peng, C.-C. Hsu, App. Therm. Eng., 88, 274-279 (2015)

10. R. Ahiska, H. Mamur, Int. J. of Renewable Energy Research, 4, 128-136 (2014)

11. D.M. Rowe, Renew. Energy, 16, 1251-1256 (1999)

12. Y.S.H. Najjar, M. Kseibi, Renew. Sust. Energ. Rev., 69, 854-861 (2017)
13. K. Sornek, M. Filipowicz, M. Zoładek, R. Kot, M. Mikrut, Energy, 166, 1303-1313 (2019)

14. G. Li, Y. Zheng, J. Hu, W. Guo, Energy, 185, 437448 (2019)

15. L.D. Landau, E.M. Lifshitz, Electrodynamics of continuous media (Pergamon Press, 1984)

16. G. Fraisse, J. Ramousse, D. Sgorlon, C. Goupil, Energy Convers. Manage., 65, 351-356 (2013)

17. F. Meng, L. Chen, F. Sun, Energy, 36, 3513-3522 (2011)

18. O.V. Marchenko, Int. J. Therm. Sci., 129, 334-342 (2018)

19. H.J. Goldsmid, Introduction to thermoelectricity, (Springer, 2010)

20. N.R. Kristiansen, G.J. Snyder, H.K. Nielsen, L. Rosendahl, J. Electron. Mater., 41, 1024-1029 (2012)

21. K. Sornek, M. Filipowicz, K. Rzepka, Energy Convers. Manage., 125, 185-193 (2016)

22. S. Khanmohammadi, M. Saadat-Targhi, A.A.A.A. Al-Rashed, M. Afrand, Energy Convers. Manage., 195, 1022-1034 (2019) 